\title{
Feature Extraction of Time-Series Data Using DWT and FFT for Ballscrew Condition Monitoring
}

\author{
Nurudeen Alegeh ${ }^{1}$, Munavar Thottoli, Naeem Mian, Andrew Longstaff, Simon \\ Fletcher \\ Centre for Precision Technologies, University of Huddersfield \\ Huddersfield, HD1 3DH, UK
}

\begin{abstract}
This paper investigates the use of the discrete wavelet transform (DWT) and Fast Fourier Transform (FFT) to improve the quality of extracted features for machine learning. The case study in this paper is detecting the health state of the ballscrew of a gantry type machine tool. For the implementation of the algorithm for feature extraction, wavelet is first applied to the data, followed by FFT and then useful features are extracted from the resultant signal. The extracted features were then used in various machine learning algorithms like decision tree, K-nearest neighbour (KNN) and support vector machine (SVM) for binary classification of the ballscrew state. The result shows significant improvement in the classification accuracy after the wavelet transform and FFT has been performed on the data.
\end{abstract}

Keywords. Ballscrew, feature extraction, Fast Fourier Transform, Discrete Wavelet Transform, machine learning.

\section{Introduction}

Feature extraction is one of the fundamental prerequisite processes used in machinelearning classification and pattern recognition applications. As such, there has been extensive research effort into the development of an accurate feature extraction technique [1-3]. Feature extraction is described as a process through which a useful set of attributes is derived from the raw time-series data while removing its redundant information. This set of attributes is also known as feature vectors [4, 5]. These features are the shorthand representation of the original raw data, which can be fed into machine learning algorithms for processing. It is important to extract features that represent the original data appropriately, otherwise, the machine-learning algorithms will not be able to process the required information accurately for classification and pattern recognition applications $[5]$.

Wavelet transformation is a technique of spectral estimation which can express general functions as an infinite series of wavelets [6]. This is achieved by shifting and scaling a mother wavelet as a linear combination of a specific set of functions or wavelet transform. The resultant decomposed signal leads to a set of coefficients referred to as wavelet coefficients. Hence, the original signal reconstruction can be achieved by a linear combination of the wavelet functions weighted by the wavelet coefficients.

\footnotetext{
${ }^{1}$ Corresponding Author. nurudeen.alegeh@hud.ac.uk
} 
The problem domain chosen for this investigation is the health of the ballscrews on a CNC machine tool. Maintaining manufacturing machines with a high degree of availability, performance and quality is of great importance wherever high-value or highthroughput manufacturing takes place. The ballscrews are one of the most critical components for the precise and accurate performance of a machine [7] since they translate the rotary motion of the motor to linear motion of the machine axis. Much work has been undertaken on the condition of motors, but very little on the transmission system.

Vibration data has been chosen as the measurable parameter to indicate ballscrew health because it is non-destructive and allows for constant monitoring devoid of unnecessary interruptions to the process [8]. The features extracted from the vibration data in conjunction with a machine-learning algorithm were used to identify and classify healthy and worn ballscrews. This is integral in high-value manufacturing applications since it will enable the users or the owners of the machine to identify and replace the ballscrews before they negatively affect the manufacturing process.

In various studies, it has been observed that the set of input data may sometimes be too large to process in most machine learning classification applications and, at times, they can also contain too much redundant information, which creates dimensionality problems for signal classification applications [4]. Processing a large amount of data also requires plenty of time and computational resources, which makes it difficult to scale up to machine - or factory - level applications. This proves that there is a significant need for reducing the dimensionality of the information. Signal processing tools such as Fourier and wavelet transforms help to reduce the amount of data that needs to be processed, while removing redundant data and retaining as much relevant information as possible, such as features, in the processed data, which represent the original data.

Cheong et al. [9] explore the use of wavelet transform for feature extraction of electroencephalogram (EEG) signals for classification. The EEG signals were obtained through a non-invasive technique using EEG electrodes. The signals represent neural activity in the brain. The authors gathered the data using relevant sensors and were able to discern the redundant frequencies in the signals. The authors categorized different frequency bands based on the frequency components the signals contain. However, they only extracted one feature (standard deviation) using a feature extraction technique employing DWT. The authors used Artificial Neural Network from MATLAB in order to train it using the feature they extracted. They were able to obtain a classification accuracy of $92.3 \%$.

Using only one feature for classification applications can lead to high uncertainty, especially in cases where the important frequency components or bands are unknown, it results in the possible misclassification of multiple signals. With multiple features, the algorithm has the opportunity to combine the result to classify signals with greater accuracy. Another aspect that must be taken into consideration is that extracting features directly from the detailed or the approximation coefficients may not necessarily yield robust features if there is a low signal to noise ratio.

Sumarno and Iswanjono [10] showed that FFT based feature extraction can be very effective in musical instrument tone identification. However, this strategy lacks robustness as it performs poorly with musical instrument tone where there are several significant peaks in the Fourier transform domain. In this paper, FFT analysis is applied in conjunction with the DWT coefficients to evaluate signals to obtain useful information that can be extracted for condition monitoring applications [11]. 


\section{Discrete wavelet transform}

The wavelet transform that is often used for feature extraction is known as Discrete Wavelet Transform (DWT). Gao and Yan [12] explain that redundancy of data in signals can be lowered during wavelet transformation by introducing discrete values of scale and translation variables. This is done by applying discretization directly on the scaling variables logarithmically, and then associate it with the step size implemented between the values of the translation variable $\tau$. This reveals hidden frequencies can be processed to obtain useful features that are highly distinguishable for machine-learning, pattern recognition and classification applications. The DWT for a signal $x(t)$ is mathematically denoted as Eq. (1):

$$
w t(j, k)=\left\langle x(t), \psi_{j, k}(t)\right\rangle=\frac{2}{\sqrt{2^{j}}} \int_{-\infty}^{\infty} x(t) \psi^{*}\left(\frac{t-k 2^{j}}{2^{j}}\right) d t
$$

Where $\psi(\mathrm{j}, \mathrm{k})(\mathrm{t})$ is the wavelet function in the time domain, $\psi^{*}$ is the complex conjugate of the wavelet function in this equation and also, $j \in Z, k \in Z$ [12].

\section{Fast Fourier Transform}

Fast Fourier Transform (FFT) converts data from the time domain to the frequency domain. It is an algorithm that converts a signal over a period of time into its frequency components. These components are the signal sinusoidal constituents of the original signal at their distinct frequency and amplitude. An FFT is basically a fast and efficient technique for computing the Discrete Fourier Transform (DFT) [10]. The DFT is mathematically represented as Eq. (2);

$$
X_{k}=\sum_{n=0}^{N-1} x_{n} e^{-i 2 \pi k n / N}, \quad k=0, \ldots, N-1
$$

Where $\mathrm{x}_{\mathrm{n}}$ represents the input series in the time domain, $\mathrm{X}_{\mathrm{k}}$ represents the output series in the frequency domain, and $\mathrm{N}$ is the number of samples. This paper uses the FFT algorithm based on the work of Frigo and Johnson [13].

\section{Experiment setup and plan}

A production 5-axis gantry machine is used for the experiments. This machine was selected because the gantry axis has two closed-loop horizontally aligned ballscrew drive mechanisms (Y1 and Y2 which are the left and right side ballscrew respectively). Y1 is in healthy condition, while Y2 has been worn from accelerated wear by restricting the normal lubrication of the ballnut. Hence, this provides a good source of experimental data to verify classification between healthy and worn ballscrew/ballnut. An accelerometer sensor (PCB piezotronics model 356A02) is located on the ballscrew as 
shown in figure 1 . The sensor is placed as close as possible to the ballnut using strong magnets. The data from the sensor is collected via a National Instruments data acquisition system. The ballscrew is covered by protective bellows to prevent damage from swarf (cutting chips), debris and dust. Fourteen experiments were conducted while analysing the "healthy" ballscrew. The axis was run at seven different nominal feed rate of 1000 , $2500,3000,5000,7500,9000$ and $1000 \mathrm{~mm} / \mathrm{min}$ in both the forward and reverse direction. These feed rates were randomly selected within the normal operating range of the machine during parts production. The same set of tests was then repeated on the "worn" ballscrew, resulting in data from 28 separate tests.

For the duration of the measurement, the gantry was made to move forward and backwards five times at a specific speed. A single vibration data set (one speed in one direction for one ballscrew) therefore contains ten vibration data samples, meaning that the captured data from the whole process is from 140 separate runs. When the vibration signal data was obtained, it was assumed that the vibrations produced by the ball screw movement will contain enough information to classify good and bad ball screws used in the machine.

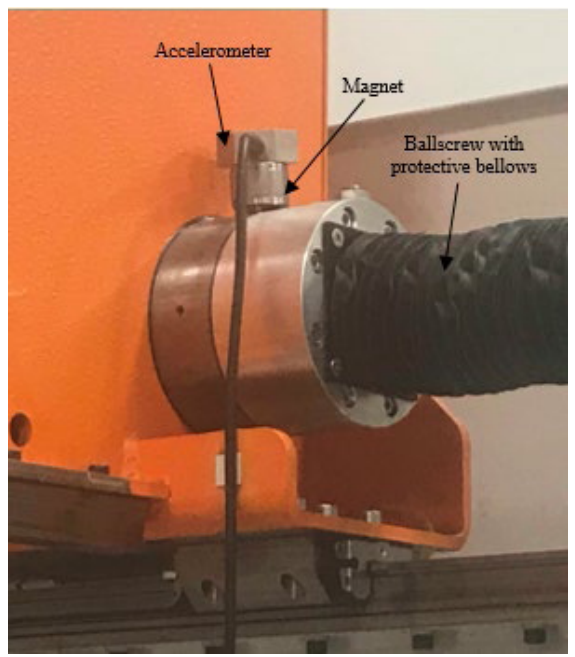

Figure 1. The experiment setup

\section{Analysis and results}

The first level Symlet 5 wavelet was the DWT decomposition used and the following features were extracted:

1. Average Magnitude; which is the arithmetic mean of the magnitudes of the frequency components from the FFT spectrum of the D1 coefficients.

2. Maximum Magnitude; which is the maximum value of the magnitude from the FFT spectrum of the D1 coefficients.

3. Minimum Magnitude; which is the minimum value of the magnitude from the FFT spectrum of the D1 coefficients.

4. Root Mean Square (RMS) of Magnitudes; which is the RMS of the magnitudes from the FFT spectrum of the D1 coefficients. 
5. Sum of Magnitudes; which is the total value of the magnitudes from the FFT spectrum of the D1 coefficients.

6. Kurtosis of Magnitudes; which is the measure of the sharpness of the magnitudes from the FFT spectrum of the D1 coefficients.

In order to compare the performance of these features extracted employing wavelet transform with the features extracted using direct statistics, the following features were extracted from the original raw data by using the traditional method without any additional processing [14, 15]: Kurtosis, Mean, Mode, Skewness, Variance. After the features were extracted, they were fed into different machine learning algorithms.

In the machine learning algorithms, $85 \%$ of the extracted features were used for training the network and $15 \%$ for validation of the trained network [16]. After training and analysis, D1 wavelet-FFT features (wavelet and FFT) is compared against statistical features, D1 Coefficient features (wavelet alone) and FFT features as shown in Figure 2. It can be observed that the wavelet-FFT features remain stable or robust across different machine learning algorithm, unlike the rest. Statistical features are a little less robust while wavelets alone appear to be the least robust with FFT alone just out-performing it. Hence the need to compliment wavelets with FFT for this application.

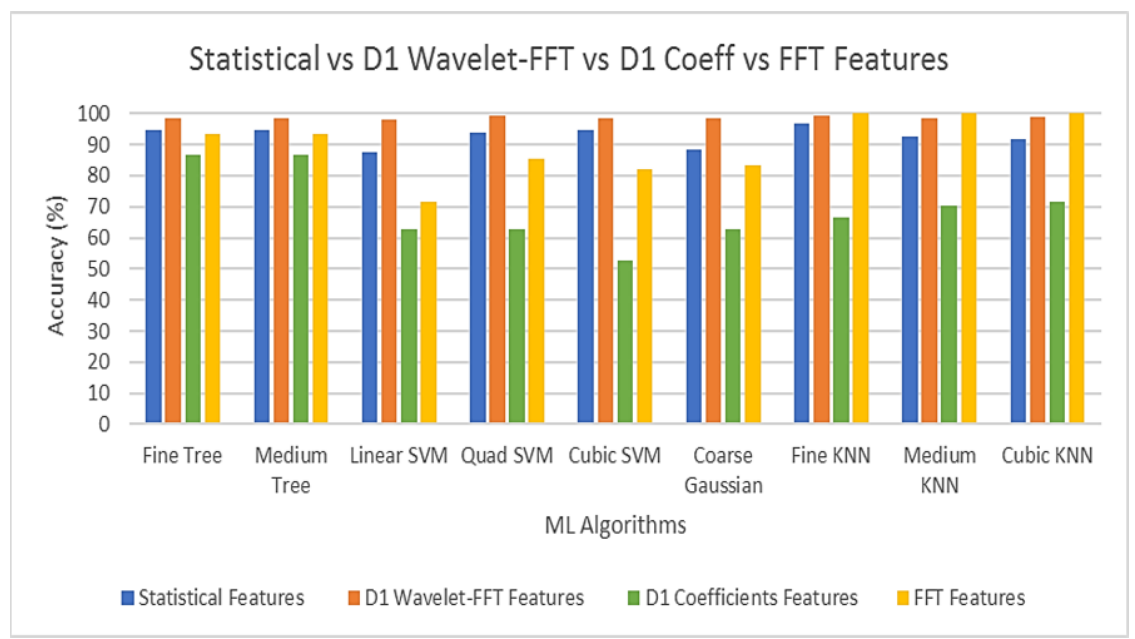

Figure 2. Bar chart of comparison among wavelet-FFT, statistical, wavelets and FFT features performance

\section{Conclusion}

This paper presents a robust method for feature extraction from time-series data using wavelets and FFT. The feature extraction method is structured into three consecutive stages: (1) wavelet decomposition to obtain the detailed coefficients; (2) FFT analysis to identify available frequencies; (3) computation of the average, minimum, maximum, RMS value, sum and kurtosis of magnitudes. The efficacy of the proposed technique is verified using data collected from the ballscrew degradation test. The achieved results indicate that the proposed method is able to accurately extract the useful features from time-series data. A comparative study showed that the proposed method performs better than the traditional method, wavelets alone and FFT alone, both in terms of accuracy and robustness. The classification accuracies for classification using wavelet-FFT features 
were above $97 \%$. The features were able to be extracted even though the information regarding the type and severity of damage sustained by the damaged ballscrews were unknown to the model.

Although this method has been applied to the classification of ballscrews health condition, future work will investigate how this can be extended to other problem domains.

\section{Acknowledgement}

The authors gratefully acknowledge the UK's Engineering and Physical Sciences Research Council (EPSRC) funding of the Future Metrology Hub (Grant Ref: EP/P006930/1) and InnovateUK and all partners of the project Servitization Project "Metrology and Digital Manufacturing for Servitisation of Manufacturing Machines" (P/N 102787).

\section{References}

[1] C. Lv et al., Feature extraction inspired by V1 in visual cortex (Ninth International Conference on Graphic and Image Processing). SPIE, 2018, p. 6.

[2] S. Woo and C. Lee, Feature extraction for deep neural networks based on decision boundaries (SPIE Defense + Security). SPIE, 2017, p. 6.

[3] M. Amiri and R. Brause, Information based universal feature extraction (Seventh International Conference on Machine Vision (ICMV 2014)). SPIE, 2015, p. 6.

[4] G. Kumar and P. K. Bhatia, "A Detailed Review of Feature Extraction in Image Processing Systems," in 2014 Fourth International Conference on Advanced Computing \& Communication Technologies, 2014, pp. 5-12.

[5] G. Borowik, J. Jankowski, and K. Kowalski, Fast algorithm for feature extraction (XXXVI Symposium on Photonics Applications in Astronomy, Communications, Industry, and High-Energy Physics Experiments (Wilga 2015)). SPIE, 2015, p. 8.

[6] A. Subasi, "EEG signal classification using wavelet feature extraction and a mixture of expert model," Expert Systems with Applications, vol. 32, no. 4, pp. 1084-1093, 2007/05/01/ 2007.

[7] M. M. Patil, "Design Calculation of Precision Ball Screw for Portable CNC Machine," International Journal for Innovative Research in Science \& Technology, Journal vol. 4, no. 1, pp. 241 - 245, 2017.

[8] I. Yesilyurt and H. Ozturk, "Tool condition monitoring in milling using vibration analysis," International Journal of Production Research, vol. 45, no. 4, pp. 1013-1028, 2007/02/15 2007.

[9] L. C. Cheong, R. Sudirman, and S. s. Hussin, Feature extraction of EEG signal using wavelet transform for autism classification. 2015, pp. 8533-8540.

[10] L. Sumarno and Iswanjono, Feature Extraction of Musical Instrument Tones using FFT and Segment Averaging. 2017, pp. 1280-1289.

[11] S. Gowid, R. Dixon, and S. Ghani, "A novel robust automated FFT-based segmentation and features selection algorithm for acoustic emission condition based monitoring systems," Applied Acoustics, vol. 88, pp. 66-74, 2015.

[12] R. X. Gao and R. Yan, Wavelets: theory and applications for manufacturing, 1st;1; ed. (no. Book, Whole). New York: Springer Science, 2010.

[13] M. Frigo and S. G. Johnson, "FFTW: an adaptive software architecture for the FFT," vol. 3, no. Conference Proceedings, pp. 1381-1384 vol.3: IEEE.

[14] G. Wang and Y. Cui, "On line tool wear monitoring based on auto associative neural network," Journal of Intelligent Manufacturing, vol. 24, no. 6, pp. 1085-1094, 2013.

[15] D. Kong, Y. Chen, and N. Li, "Gaussian process regression for tool wear prediction," Mechanical Systems and Signal Processing, vol. 104, pp. 556-574, 2018.

[16] A. Salimiasl and A. Özdemir, "Analyzing the performance of artificial neural network (ANN)-, fuzzy logic (FL)-, and least square (LS)-based models for online tool condition monitoring," The International Journal of Advanced Manufacturing Technology, vol. 87, no. 1, pp. 1145-1158, 2016. 